\title{
A method for fixing and paraffin embedding tissue to retain the natural fluorescence of reporter proteins
}

\section{Protocol For: \\ A method for fixing and paraffin embedding tissue to retain the natural fluorescence of reporter proteins}

Akifumi Nakagawa, Kate Von Alt, Keith D. Lillemoe, Carlos Fernández-del Castillo, Andrew L. Warshaw, and Andrew S. Liss

Department of Surgery and the Andrew L Warshaw, MD, Institute for Pancreatic Cancer Research, Massachusetts General Hospital and Harvard Medical School, Boston, MA

BioTechniques Protocol Guide, January 2017; doi 10.2144/000114505

See full protocol online: https://benchtalk.biotechniques.com/users/8521-biotechniques-editors/posts/3966-protocol-for-a-method-for-fixing-andparaffin-embedding-tissue-to-retain-the-natural-fluorescence-of-reporter-proteins

Protocol Summary: Here we present an ethanol fixation protocol that allows for the direct visualization of the natural fluorescence of reporter proteins while maintaining excellent tissue histology. We demonstrate the utility of this method for visualizing green and red fluorescent proteins in a wide range of murine tissues using both cytoplasmic and membrane-localized fluorescent reporter proteins. Tissues fixed by this method also allow for immunohistochemical studies, providing a single method to visualize the natural fluorescence of reporter proteins with subsequent detection of cellular proteins.

A
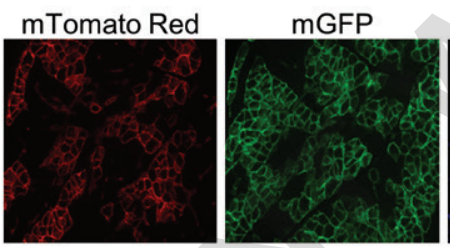

B

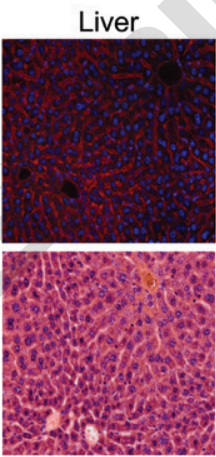

DAPI
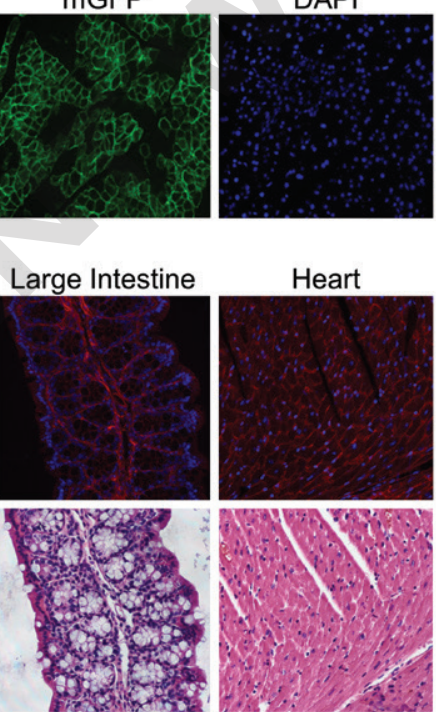

Heart
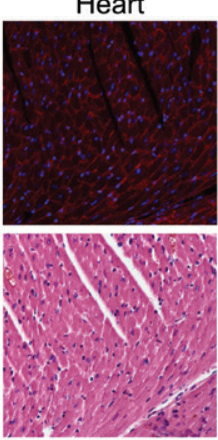

Merge

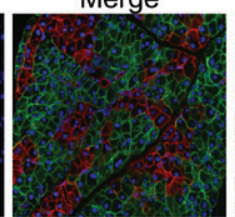

Kidney

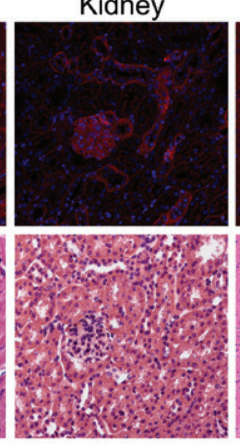

IHC GFP

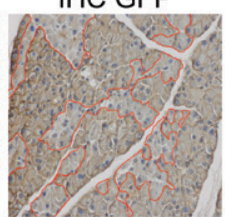

Brain

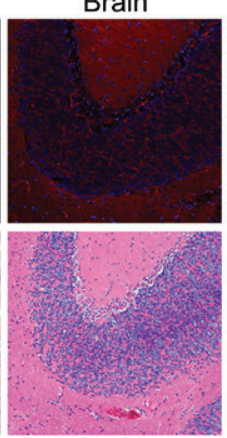

Protocol Schematic: Natural fluorescence of membrane-localized reporter proteins. (A) Representative images from pancreas of Pdx1-cre;mTmG mice. Red and green fluorescence of membrane-localized reporter proteins as well as DAPI-stained nuclei (blue) are shown. Matched image from slide that was processed for GFP immunohistochemistry $(\mathrm{IHC})$ is shown on the right. Areas of tissue that do not express GFP but do express $\mathrm{mT}$ are outlined in red. (B) Representative fluorescence images of $\mathrm{mT}$ and DAPI-stained nuclei in the liver, large intestine, heart, kidney, and brain of Pdx1-cre;mTmG mice. Matched hematoxylin and eosin (H\&E)-stained images are shown in the bottom row. 